\section{Double decompression of presacral collection by heald anal stent and foley catheter combination}

\author{
Dean A. Godfrey, Guy F. Nash \\ Department of Surgery, Poole Hospital, \\ Poole, Dorset, UK
}

\begin{abstract}
The authors demonstrate the effective drainage of presacral sepsis following low anterior resection surgery using the novel approach of both the Heald anal stent and a Foley catheter combined. The Heald stent was placed trans-anally without the need for anaesthetic and an 18Fr Foley was placed through this. A presacral collection was drained through the stent, allowing the patient to be discharged after a week without the need to return to the operating theatre. The Heald stent may be used to successfully drain a presacral collection by double decompression of the rectum and the presacral space
\end{abstract}

\section{Introduction}

The use of the Heald anal stent has previously been described in the successful therapeutic decompression of the rectum following a leaking ileorectal anastomosis ${ }^{1}$. The novel technique of using a Heald stent in combination with Foley catheter to drain a presacral collection following a leak from rectal cancer surgery is presented.

\section{Case Report}

A 65-year-old man presented with rectal bleeding and was diagnosed with a rectal cancer $8 \mathrm{~cm}$ above the sphincter. Biopsy and cross sectional imaging was suggestive of early T3N0M0 adenocarcinoma- subsequently confirmed on histology. The patient had a background of radical radiotherapy in 2005 for prostatic adenocarcinoma (T2N0) in addition to ischaemic heart disease, warranting percutaneous coronary intervention and insertion of a drug eluting stent for single vessel disease in 2008 . The patient remained well following these interventions and surveillance biochemistry has been reassuring. Similarly, his function was unlimited in relation to his co-morbidities and therefore con- sidered an intermediate risk candidate for curative resectional surgery.

He underwent a low laparoscopically assisted anterior resection with total mesorectal excision and stapled anastamosis $4 \mathrm{~cm}$ above the pelvic floor. As the leak rate following radiotherapy in this field is considerable, a defunctioning loop ileostomy was planned. Intra-operative drain insertion is not common local practice and with pre-operative bowel preparation, we attempt to minimize anastamotic strain to both mechanical and chemical factors. Despite this, the patient developed a large presacral collection (Figure 1) a week following surgery from a small posterior anastamotic leak. This was calculated to measure $60 \times 50 \times 50 \mathrm{~mm}$ (in craniocaudal, transverse and anteroposterior planes respectively).

The patient became septic without peritonism and drainage techniques were considered. In our unit, we have had prior experience of using a Heald stent in the successful drainage of pelvic collections following colorectal resectional surgery. Following discussion with our radiology colleagues, it was decided the combination of Foley catheter drainage (i.e. placed in the presacral collection) via a transanal approach would be suitable (Figure 2). This combination allows double decompression, as if the Heald stent were not used, the positive rectal pressure may encourage further contamination via the non healed anastamotic line. It was also considered to have fewer potential complications and be rather easier to manage from a nursing viewpoint (in terms of patient positioning and wound site dressing) than a percutaneously placed radiological drainage technique.

Following informed consent, a stent was inserted trans-anally on the ward after instilling local anaesthetic jelly (i.e. without the need for general or percutaneous anaesthesia). Through the trans-anally placed Heald stent, a 18Fr Foley catheter was directed through the unhealed anastamotic line into the presacral space and the balloon inflated to $5 \mathrm{~mL}$ (with water) to hold its position. The catheter was then flushed with $20 \mathrm{~mL}$ betadine (povidone iodine). The procedure was tolerated exceptionally well with only minor intra and post-procedural discomfort alleviated with simple analgesia and reassurance.

\section{Results}

Over the next week, the drainage of the stent continued as the patients septic markers normalised. On the fifth day a magnetic resonance imaging (MRI) pelvis (Figure 3) confirmed that the collection had almost
Correspondence: Guy F. Nash, Department of Surgery, Poole Hospital, Poole, Dorset, BH15 2JB, UK.

Tel. +44.120.244.8798 - Fax: +44.120.244.2615

E-mail: guy.nash@poole.nhs.uk

Key words: rectal cancer, surgery, sepsis, stent.

Received for publication: 18 June 2011

Accepted for publication: 21 October 2011.

This work is licensed under a Creative Commons Attribution NonCommercial 3.0 License (CC BYNC 3.0).

(c) Copyright D. A. Godfrey and G.F. Nash., 2011 Licensee PAGEPress, Italy

Surgical Techniques Development 2011; 1:e28 doi:10.4081/std.2011.e28

drained and the stent was painlessly removed on the ward. The patient was discharged the following day and avoided the need for further intervention until loop ileostomy reversal 6 months subsequently.

\section{Discussion}

Pelvic sepsis is more common following neoadjuvant radiotherapy and particularly

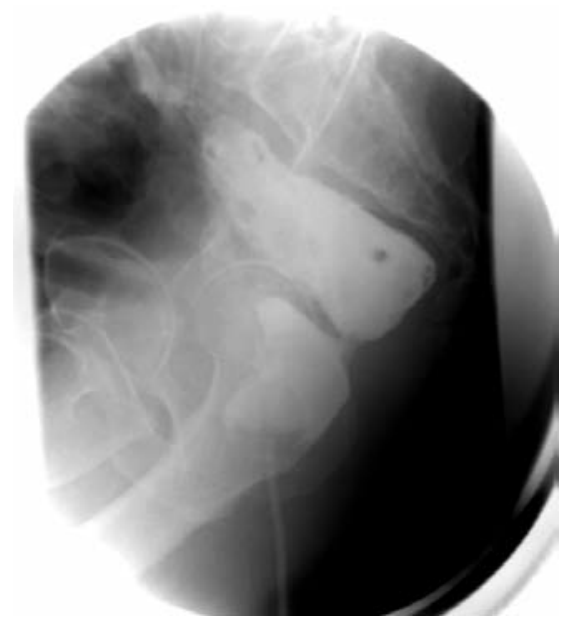

Figure 1. Contrast enema demonstrating fluid collection in presacral space.

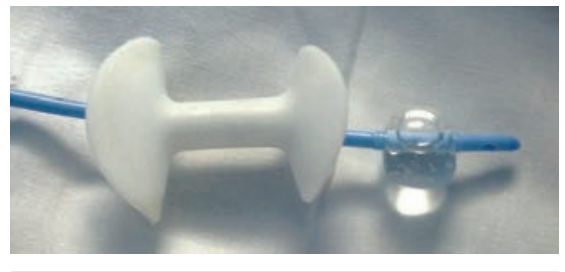

Figure 2. Heald stent and Foley combination drain. 


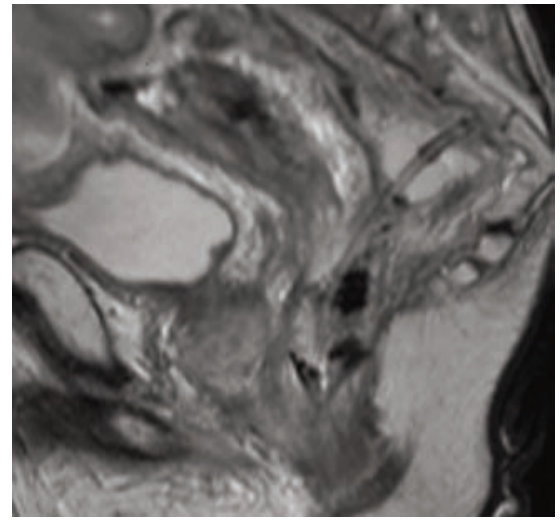

Figure 3. Sagittal magnetic resonance imaging of pelvis $[A=$ anterior, $P=$ posterior] five days after the insertion of the Heald stent. Urinary catheter shown in the presacral collection, now decompressed. when the patient has an anastomosis ${ }^{2}$. The Heald stent allows the entry of air to inhibit anaerobic bacterial growth. The Foley cather should be of large bore and irrigation can be carried out if necessary. The Heald stent has been previously demonstrated to be an alternative, though not foolproof, method of avoiding a defunctioning loop stoma in low rectal anastomoses ${ }^{3}$, however is still used routinely after high anterior resections at some centers. We recommend this technique of allowing free rectal luminal drainage via the Heald stent in combination with a Foley catheter to allow presacral drainage of postop collections at the site of low colorectal anastamoses. This combination allows double decompression, if the Heald stent were not used the positive rectal pressure may encourage further contamination via the non-healed anastomtic line.

\section{References}

1. Brent A, Armstrong T, Nash GF, Heald RJ. Therapeutic use of the Heald Silastic Anal Stent. Colorectal Dis 2007;9:279-80.

2. Buie WD, MacLean AR, Attard JA, et al. Neoadjuvant chemoradiation increases the risk of pelvic sepsis after radical excision of rectal cancer. Dis Colon Rectum 2005;48:1868-74.

3. Amin AI, Ramalingam T, Sexton R, et al. Comparison of transanal stent with defunctioning stoma in low anterior resection for rectal cancer. Br J Surg 2003;5: 581-2. 